\title{
Variation of Solar Wind Parameters During Intense Geomagnetic Storms
}

\author{
Ayush Subedi, Binod Adhikari and Roshan Kumar Mishra \\ Department of Physics, St. Xavier College, Maitighar, Kathmandu. \\ Email: roshanmishra.phy@gmail.com
}

\begin{abstract}
Geomagnetic disturbances are caused by enhanced solar wind magnetospheric energy coupling process. The principal cause of geomagnetic disturbance is the magnetic reconnection that establishes an electrodynamical coupling between the solar wind plasma and magnetosphere. Around solar maximum, the main structures emanating from the sun are sporadic Coronal Mass Ejection (CMEs) and their interplanetary counterparts (ICMEs). During the descending and minimum solar cycle phases, coronal holes occur more often. They appear as dark regions confined to Solar poles during the solar maximum but expand in size and moves toward the solar equator during the descending phase. In this work, we have taken three different geomagnetic storms during solar maxima. For the interpretation of events, we used interplanetary solar wind data and geomagnetic indices. These satellite data and Dst indices (ranging from -100nT to above) are interpreted by using the method of cross correlation. The values of Bz found approximately 20nT, -50nT and $-20 n T$ respectively. Similarly, the value of Dst is $-250 n T,-400 n T$ and $-300 n T$ which shows very intense effect. Likewise, the correlation coefficient we obtained from this research work strongly suggest that interplanetary magnetic field Bz has strong impact for the cause of geomagnetic storms.
\end{abstract}

Keywords: Geomagnetic storms, Solar wind, Cross-correlation analysis

\section{INTRODUCTION}

Astream of energized containing charged particles, primarily electrons and protons, flowing outward from the Sun, through the solar system at speed of about $400 \mathrm{~km} / \mathrm{s}$ and at a temperature of 1 million degrees (Celsius), which is made of plasma, is called solar wind (Parker, 1958). Approximately 100 tons of solar wind is releasing per day from the sun. It contains large amount of kinetic and electrical energy. Some amount of these energies directly enters into the earth's magnetosphere and due to which the earth is heated. When these energies enter inside the magnetosphere it also creates turmoil to the geomagnetic activity which ultimately results to the geomagnetic storms, sub-storms and aurora (Chapman, Bartels 1962, Gonzalez et.al, 1994).

The temporary disturbance created by the solar wind after entering inside the earth's magnetosphere when the magnetic field of solar wind interacts with earth's magnetic field (Dungey 1961). Initially the earth's magnetosphere is compressed when the solar wind pressure is increased. During the time of interaction between the earth's magnetic field and solar wind magnetic field certain amount of energy is increased and this increased energy ultimately increase the plasma level in magnetosphere and also increase the movement of electric current in magnetosphere and ionosphere (Dungey, 1961; Gonzalez et al., 1994). The geomagnetic storms are classified into the four categories as per the value of Dst as suggested as weak/small (-30nT to $-50 \mathrm{nT})$; moderate $(-50 \mathrm{nT}$ to $-100 \mathrm{nT})$; intense $(-100 \mathrm{nT}$ to $-250 \mathrm{nT})$ and very intense (-250nT an above) (Gonzalez.et.al; 1994). Geomagnetic activity also creates some directly and indirectly effect and among those some are health problems, satellite malfunction, weather changes etc. Similarly, auroras are produced when the solar wind extremely disturb the magnetosphere and the trajectories of charged particles in both solar wind and magnetosphere plasma, mainly in the form of electrons and protons precipitate them in the upper atmosphere where their energy is lost. Simply when the charged particles from the sun strikes to the upper atmosphere it results to the colored light in the sky which is known to be aurora (Tsurutani; Gonzalez, 1997).

Those phenomena which occur within the magnetically heated outer atmospheres in the sun are known to be solar phenomena, which includes solar wind, energy bursts such as solar flares, coronal mass ejection or solar eruptions, coronal heating and sunspots (Camp; Tung, 2007). All these phenomena are the results of a helical dynamo near the center 
of the sun's mass which generates strong magnetic field and a chaotic dynamo near the surface which generates smaller magnetic field fluctuations (Kivelson; Russel, 1995). The sum of these all solar fluctuations is termed as solar variation and these collective effects of solar variations within sun's gravitational field is termed as space weather. Solar minima and solar maxima are the period which falls under the solar activity. The period of least solar activity in the 11 year solar cycle of the sun is called as solar minima and during the time of solar minima sunspot and solar flare activity diminishes and sometime does not occur for several days. The solar minima can only identify after the six month of the actual occurrence of solar minima because the minimum is described by a smoothed average over 12 months of sunspot activity. The normal period of the greatest solar activity in the 11 year solar cycle of the sun is called as solar maxima. At this time the large numbers of sunspots appear and the sun's irradiance output grows by about $0.07 \%$, which directly impact the earth's global climate (Burlaga,

1995; Harvey et al., 2000).

All the geomagnetic disturbances occurring over short periods of time are measured by geomagnetic indices. It was found that the magnetic field measured near the earth surface varies in wide ranges. The main cause for geomagnetic field variations are the solar wind coupling with the earth's magnetosphere and magnetosphere coupling with ionosphere (Dungey, 1961; Gonzalae et al., 1994) The main indices used to measure variations in the earth's magnetic field strength are- Dst index and AE index (Sugiura, 1964; Rostoker, 1972). The short time disturbance (Dst) index is the index which is used to monitor the worldwide magnetic storm level. It is constructed by averaging $\mathrm{H}$ from midlatitude and equatorial magneto grams from all over the world. The values of the Dst index is negative which means that a magnetic storm is in progress. This is due to the storm time ring current which flows around the earth from east to west in the equatorial plane. The more negative value of Dst the more the intensity of the magnetic storm. The ranges of Dst values depends upon the activity of the sun. The approximated ranges of Dst recorded are $+100 \mathrm{nT}$ to $-600 \mathrm{nT}$. The positive value of the Dst is due to the compression of magnetosphere due to increase in solar wind pressure. The global auroral zone magnetic activity produced by enhanced ionospheric current is measured by AE (auroral electro jet) index (Rostoker, 1972; Kivelson; Russel, 1995). Electro jet is the electric current which travels around the D and $\mathrm{E}$ region of the ionosphere. It is constructed by taking one minute datasets of H-components from auroral zone observatories located around the world. So the constructed values are plotted as a function of universal time (UT). The amplitude of upper and lower envelopes is defined as AU and AL respectively. The amplitude of AU represents the maximum perturbation generated by eastward electro jet current in the afternoon sector and AL represents the maximum perturbation generated by westward electro jet current in the morning and midnight sectors. The difference between $\mathrm{AU}-\mathrm{AL}$ gives the $\mathrm{AE}$ index and $(A U+A L) / 2$ give the $\mathrm{AO}$ index. In general, the term "AE" is used to indicate $\mathrm{AU}, \mathrm{AL}, \mathrm{AE}$ and $\mathrm{AO}$.

\section{DATA SET AND METHODOLOGY}

We used internet based supply of data provided by the International space related research centers that is OMNI (Operating Mission as Nodes on the Internet web system) (http://omniweb.gsfc.nasa.gov/ow.html). The OMNI system has been conducting its research using many ground and space based GPS and satellite stations since long ago. The output product of their observation and research work are kept in the website for benefit of people like us. We investigate 3 different intense geomagnetic storm and study the variation of solar wind parameters during intense geomagnetic storms at a particular time. We used cross correlation between $\mathrm{Bz}$ (possible cause) with several solar wind parameters (consequence) by using satellite data. This analysis allows obtaining the degree of correlation and also the lag between the two series. This lag is very important because it gives us the response time of the phenomenon and can give us an idea of the mechanism operating in the energy transfer.

\section{RESULTS AND DISCUSSION}

\section{Case1: Geomagnetic storm occurred in 24 November 2001}

Figure 1 represents the OMNI datasets during 24 November 2001. In this figure, the geomagnetic indices present very intense value. During the main phase, the temperature $\mathrm{T}$ 
(10e4k) is nearly about $190 \mathrm{~K}$, plasma speed (Vsw) is around $1000 \mathrm{~km} / \mathrm{s}$, density $\mathrm{D}(\mathrm{n} / \mathrm{cc})$ is about 50n/cc. Similarly, the value of Bz shows -20 nT; Dst index shows around -250 nT and the AE index shows below $1800 \mathrm{nT}$. Thus the solar wind parameters, interplanetary magnetic field and components show intense values. During a geomagnetic disturbance, there is an energy input inside the magnetosphere and ionosphere which changes ionospheric parameters, such as composition, temperature and circulation (Gonzalez et.al, 1994); Gonzalez et.al, 1999).
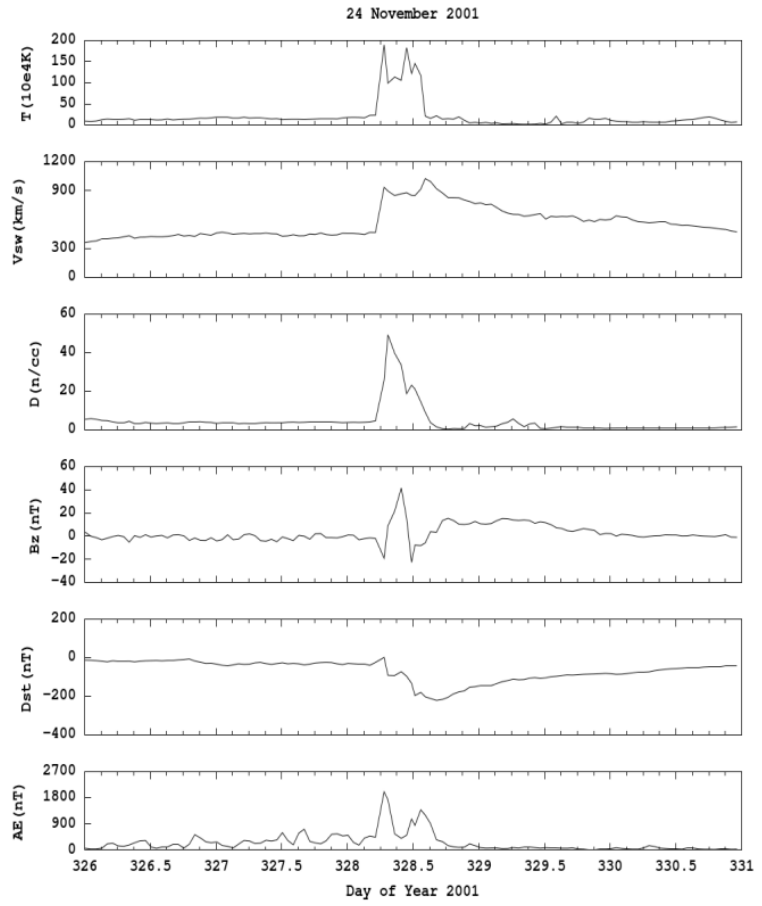

Figure 1. From top to bottom, the panels shows the variations of solar wind temperature (T), solar wind velocity (Vsw), solar wind density ( $\mathrm{n} / \mathrm{cc}$ ), the south-north component of magnetic field $\mathrm{Bz}$ (nT) in GSM coordinate system, Dst (nT) and AE (nT) indices with time (days) respectively for the case-1, 24 November 2001.

Figure 2 gives the cross-correlation results of $\mathrm{Bz}$ with Vsw, n(cc), Dst, and AE index during 24 November 2001. The horizontal axis represents the scale in minute and the vertical axis represents the cross-correlation coefficient. In this figure, the scales of $-150,-100,-50,0,50,100$ and 150 are labeled in the horizontal axis and cross-correlation coefficient runs to it range in the vertical axis. In this figure, $\mathrm{Bz}$ and Vsw shows a positive correlation coefficient of about 0.4 at zero time lag. Similarly, Bz and solar wind density (den) shows a positive correlation coefficient of about 0.5 at time lag of 25 minutes; $\mathrm{Bz}$ and Dst shows a negative correlation coefficient of about 0.6 at time lag of 5 minutes; $\mathrm{Bz}$ and $\mathrm{AE}$ shows a positive correlation coefficient of about 0.55 at time lag of 25 time minutes.

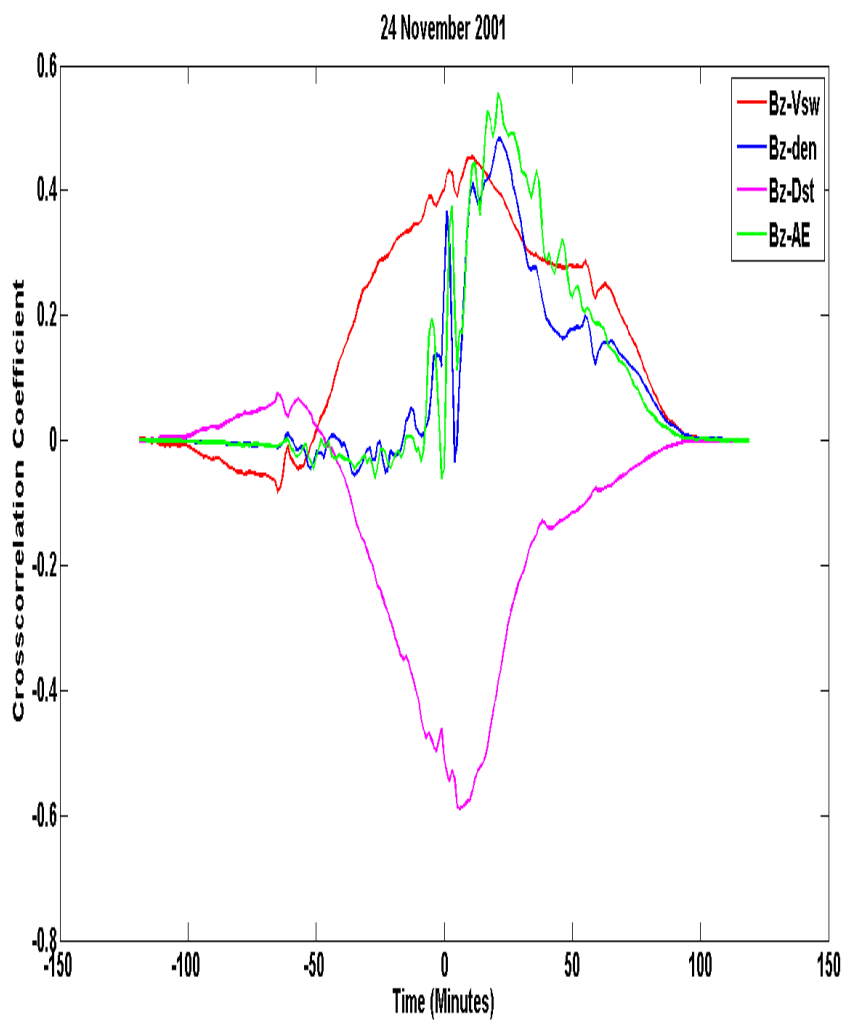

Figure 2. Cross-correlation of south-north component of magnetic field, Bz (nT) in GSM Coordinate with (Vsw, den, Dst and AE) index during 24 November 2001.

\section{Case2: Geomagnetic storm occurred in 31 March 2001}

Figure 3 represents the OMNI datasets during 31 March 2001. In this figure, the geomagnetic indices is in intense value. During the main phase, the temperature $\mathrm{T}(10 \mathrm{e} 4 \mathrm{k})$ is nearly about $70 \mathrm{~K}$, plasma speed (Vsw) is around 850 $\mathrm{km} / \mathrm{s}$, density $\mathrm{D}(\mathrm{n} / \mathrm{cc})$ is about $40 \mathrm{n} / \mathrm{cc}$. Similarly, the value of Bz shows -50 nT; Dst index shows around $-400 \mathrm{nT}$ and the AE index shows below $1500 \mathrm{nT}$. Thus the solar wind parameters, interplanetary magnetic field and components show very intense values (Adhikari, 2015). 

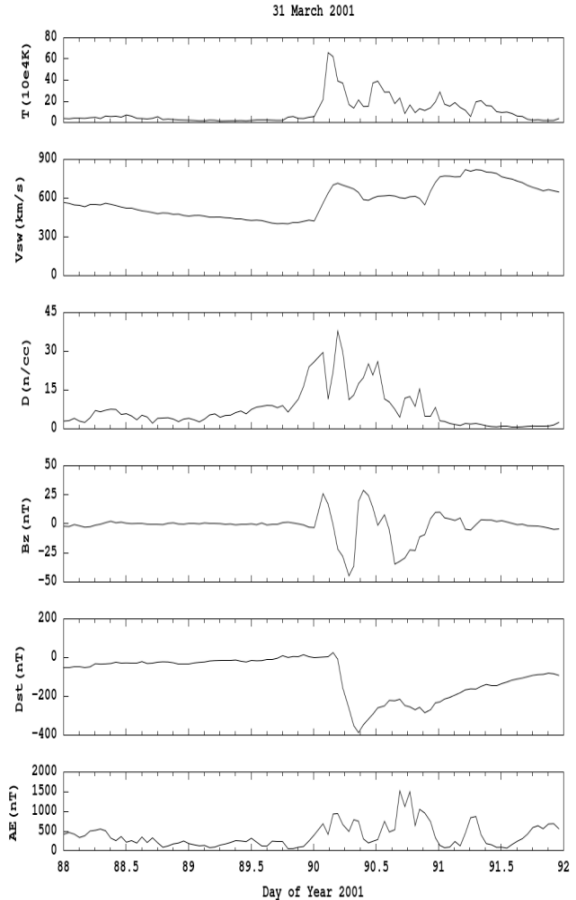

Figure 3. From top to bottom, the panels shows the variations of solar wind temperature (T), solar wind velocity (Vsw), solar wind density (n/cc), the south-north component of magnetic field Bz $(n T)$ in GSM coordinate system, Dst (nT) and AE (nT) indices with time (days) respectively for the case-2, 31 March 2001.

Figure 4 gives the cross-correlation results of $\mathrm{Bz}$ with (Vsw, den, Dst, AE) index during 31 March 2001. The horizontal axis represents the scale in minute and the vertical axis is similar as in figure 5. In this event, only BZ-AE shows correlation of about 0.5 at zero time lag.

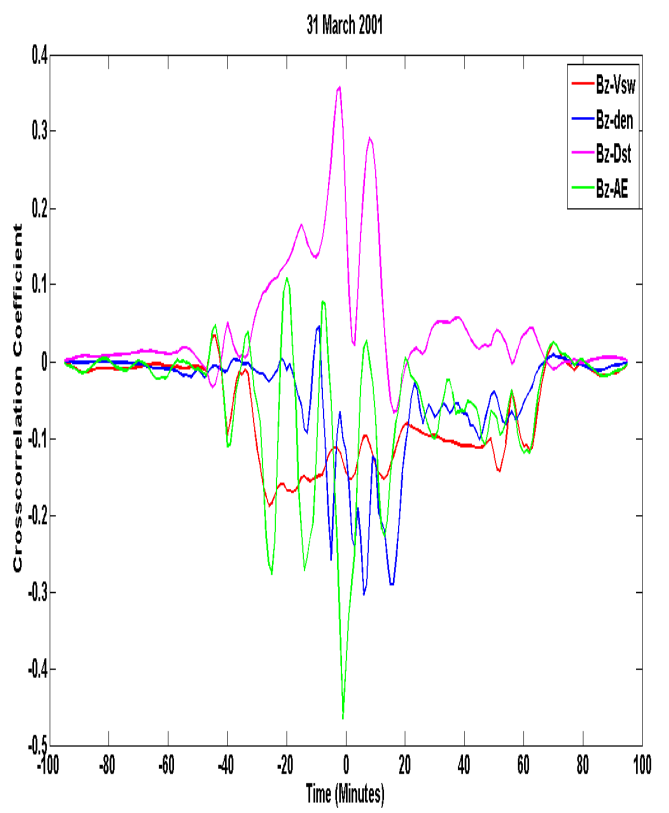

Figure 4. Cross-correlation of south-north component of magnetic field, $B z(n T)$ in GSM Coordinate with (Vsw, den, Dst and AE) index during the 31 March 2001

\section{Case3: Geomagnetic storm occurred in 11 April 2001}

Figure 5 represents the OMNI datasets during the case-3, 11 April 2001. In this figure, the geomagnetic indices is in very intense value. During the main phase, the temperature $\mathrm{T}$ $(10 \mathrm{e} 4 \mathrm{k})$ is nearly about $70 \mathrm{~K}$, plasma speed $(\mathrm{Vsw})$ is around $850 \mathrm{~km} / \mathrm{s}$, density D (n/cc) is about $25 \mathrm{n} / \mathrm{cc}$. Similarly, the value of Bz shows -20 nT; Dst index shows around -300 nT and the $\mathrm{AE}$ index shows below $1750 \mathrm{nT}$. This events also shows very intense values for all parameters and indiecs.

11 April 2001
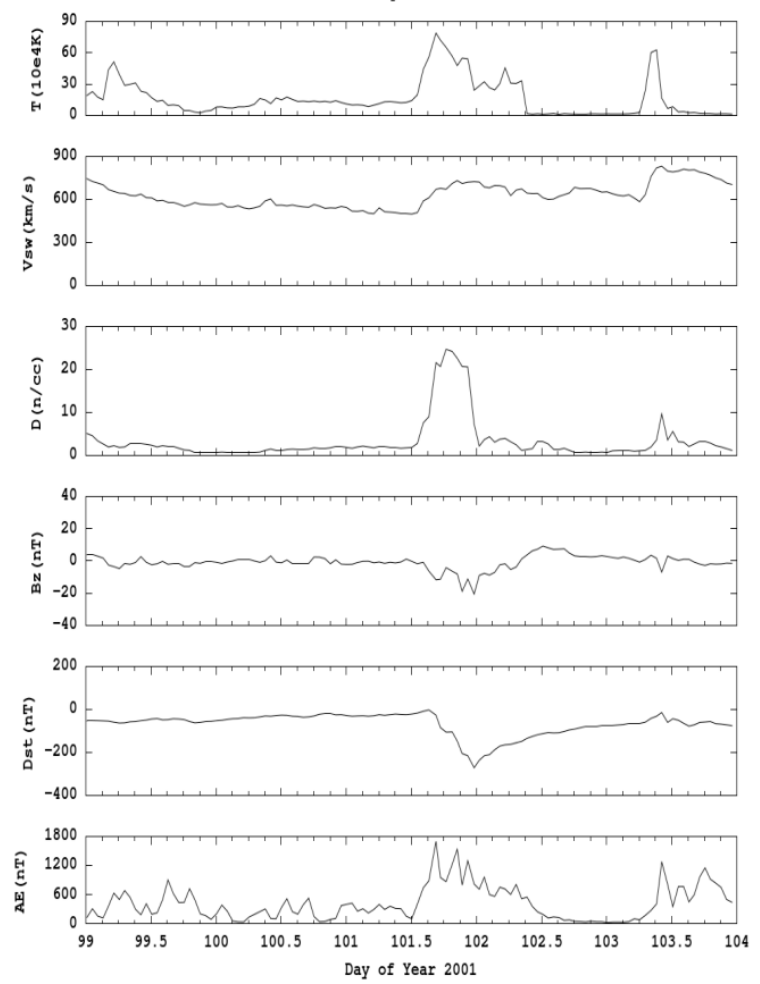

Figure 5. From top to bottom, the panels shows the variations of solar wind temperature (T), solar wind velocity (Vsw), solar wind density $(\mathrm{n} / \mathrm{cc})$, the south-north component of magnetic field Bz (nT) in GSM coordinate system, Dst (nT) and AE (nT) indices with time (days) respectively for the event-3, 11 April 2001

Figure 6 gives the cross-correlation results of Bz with (Vsw, den, Dst, AE) index during 11 April 2001. The horizontal axis and vertical axis are similar as in figure 4. In this figure, $\mathrm{Bz}$ and Vsw shows a positive correlation coefficient of about 0.8 at zero time lag; Bz and den shows a positive correlation coefficient of about 0.7 at zero time lag; Bz and Dst shows a positive correlation coefficient of about 0.8 at zero time lag; 
$\mathrm{Bz}$ and $\mathrm{AE}$ shows a negative correlation coefficient of about 0.9 at zero time lag.

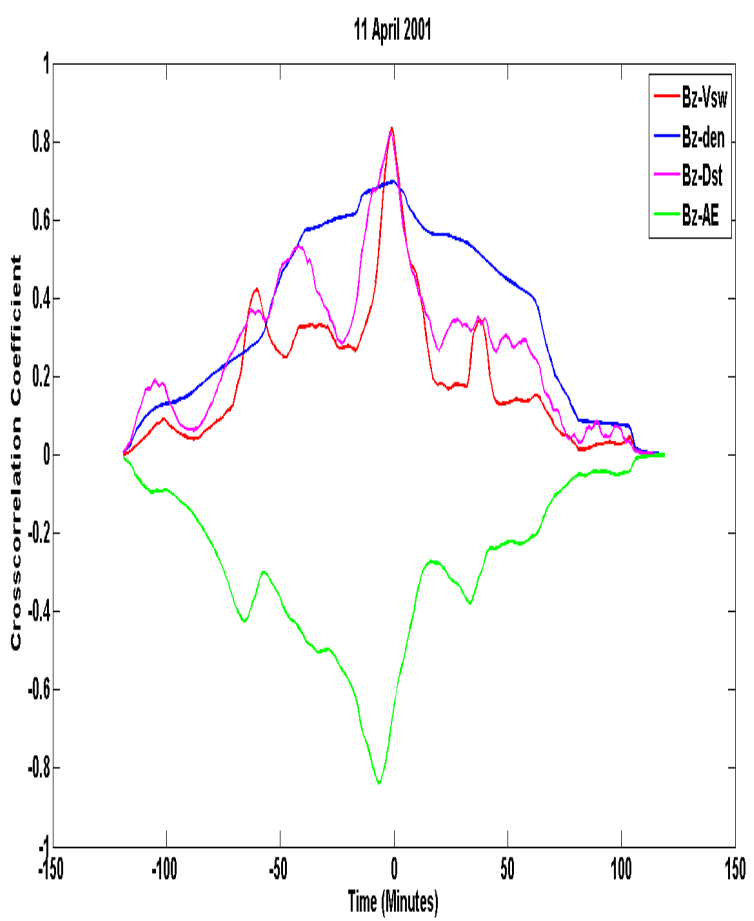

Figure 6. Cross-correlation of south-north component of magnetic field, Bz (nT) in GSM Coordinate with (Vsw, den, Dst and AE) index during the Event-3, 11 April 2001.

\section{CONCLUSION}

The study of geomagnetic storm is an important issue for the study of space weather. The $\mathrm{Bz}$ component is an important parameter which indicates the presence of geomagnetic storm. In this research, we study several solar wind parameters and geomagnetic indices. We use cross correlation analysis of Bz-component with several parameters. In this research work the variation of different parameters during geomagnetic storms was studied. For this purpose, we select the three different events. These three events are quite different from each other and also give the different results. The cross-correlation and the variations of different parameters were obtained. The Bz was found to be approximately $-20 \mathrm{nT}$, $-50 \mathrm{nT}$, and $-20 \mathrm{nT}$ in events 1,2 and 3 respectively.

From our study, we observe that case 1, 2 and 3 show very intense effect described on the basis of the Gonzalez et al. (1994) and have the Bz value $-20 \mathrm{nT},-50 \mathrm{nT}$ and $-20 \mathrm{nT}$ respectively. Similarly, the correlation coefficient we obtained from this research work strongly suggest that the interplanetary magnetic field $\mathrm{Bz}$ has strong impact for the cause of geomagnetic disturbances.

\section{REFERENCES}

1) Adhikari, B. HILDCAA-Related Effects Recorded in Middle Low Latitude Magnetometers. Institution Nacional de Pesquisas Espaciais, Phd thesis, Sao Jose dos Campos, Brasil, (2015).

2) Burlaga, L. F. Interplanetary magnetohydrodynamics. In: Interplanetary Magnetohydrodynamics. Oxford Univ. Press, New York, (1995).

3) Camp, C. D.; Tung, K. K. Surface warming by the solar cycle as revealed by the composite mean difference projection. Geophysical Research Letters, 34, (2007).

4) Chapman, S.; Bartels, J. Geomagnetism. Clarendon Press, Oxford, (1962).

5) Dungey, J. W. Interplanetary magnetic field and the auroral zones. Physical Review Letters, 6, 47-48, (1961).

6) Echer, E.; Alves, M. V. and Gonzalez W. D. Geoeffectiveness of Interplanetary Shocks during Solar Minimum (1995-1996) and Solar Maximum (2000). Solar Physics, 221, 361-380, (2004).

7) Firoz, K. A. Cosmic Rays and Space Weather. Ph.D. Thesis, Institute of Physics, University of Pavol Jozef Safarik, Slovak Republic (2008).

8) Gonzalez, W. D.; Josely, J. A.; Kamide, Y.; Korehi, H. W.; Rostoker, G.; Tsuruntani, B. T.; Vasylianas V. M. What is a geomagnetic storm qm? Journal of Geophysical Research, 99, 5771-5792, (1994).

9) Gonzalez, W. D.; Tsurutani, B. T.; Clua-Gonzalez, A. L. Interplanetary origin of geomagnetic storms. Space Science Reviews, 88, 529-562, (1999).

10) Harvey, K.; Suess, S.; Aschwanden, M.; Guhathakurta, M.; Harvey, J.; Hathaway, D.; Labonte, B.; Sheeley, N.; Tsurutani, B. T. A NASA workshop on coronal holes near solar maximum and over the solar cycle. In: Washington: NASA: (2000).

11) Kivelson, M. G.; Russel, C. T. Introduction to Space Physics. Cambridge University Press, (1995).

12) Mayaud, P. N. Derivation meaning and use of geomagnetic indices. Union, 22, Washington DC, 
Geophysics. Monogr. Am Geophys, 154, 34-36, (1980).

13) Parker, E. N. Dynamics of the interplanetary gas and magnetic fields. Astrophysical Journal, 128, 664, (1958).

14) Rostoker, G. Geomagnetic indices. Reviews of Geophysics and Space Physics. 10, 935-950, (1972).

15) Rathore, B. S.; Kaushik, S. C.; Firoz, K. A.; Gupta, D. C.; Shrivastva, A. K.; Parashar, K. K.; Bhadoria, R. M. A Correlative Study of Geomagnetic Storms Associated with Solar Wind and IMF Features During Solar Cycle-23 (2011). International Journal of Applied Physics and Mathematics, 1, 149-154.

16) Sugiura, M. Hourly values of equatorial Dst for the IGY. Annals of the International Geophysical Year, 35, 9, (1964).

17) Tsurutani, B. T.; Gonzalez, W. D. The interplanetary causes of magnetic storms. A review, in Magnetic Storms, AGU monograph, 98, 77, (1997). 